\title{
Gender differences of patients at-risk for psychosis regarding symptomatology, drug use, comorbidity and functioning - Results from the EU-GEI study
}

\author{
Stephanie Menghini-Müller ${ }^{\mathrm{a}, \mathrm{b}}$, Erich Studerus ${ }^{\mathrm{a}}$, Sarah Ittig ${ }^{\mathrm{a}}$, Ulrike Heitz ${ }^{\mathrm{a}}$, \\ Laura Egloff $^{\mathrm{a}}$, Christina Andreou ${ }^{\mathrm{a}}$, Lucia R. Valmaggia ${ }^{\mathrm{d}}$, Matthew J. Kempton ${ }^{\mathrm{c}}$, \\ Mark van der Gaag, ${ }^{\mathrm{e}, \mathrm{f}}$, Lieuwe de Haan ${ }^{\mathrm{g}, \mathrm{h}}$, Barnaby Nelson ${ }^{\mathrm{i}}$, \\ Neus Barrantes-Vidal ${ }^{\mathrm{j}}$, Merete Nordentoft ${ }^{\mathrm{k}, \mathrm{l}}$, Stephan Ruhrmann ${ }^{\mathrm{m}}$, \\ Gabriele Sachs $^{\mathrm{n}}$, Bart P. Rutten ${ }^{\mathrm{o}}$, Jim van Os ${ }^{\mathrm{p}, \mathrm{q}, \mathrm{r}}$, Anita Riecher-Rössler ${ }^{\mathrm{a}, *}$, \\ EU-GEI High Risk Study Group ${ }^{1}$
}

a Center for Gender Research and Early Detection, University of Basel Psychiatric Hospital, Basel, Switzerland

${ }^{\mathrm{b}}$ University of Basel, Department of Psychology, Division of Clinical Psychology and Epidemiology, Basel, Switzerland

${ }^{\mathrm{c}}$ Department of Psychosis Studies, Institute of Psychiatry, Psychology E' Neuroscience, King's College London, London, United Kingdom

d Department of Psychology, Institute of Psychiatry, Psychology E Neuroscience, King's College London, London, United Kingdom

${ }^{\mathrm{e}}$ VU University, Faculty of Behavioural and Movement Sciences, Department of Clinical Psychology and EMGO+ Institute for Health Care Research,

Amsterdam, the Netherlands

${ }^{\mathrm{f}}$ Parnassia Psychiatric Institute, Department of Psychosis Research, The Hague, the Netherlands

${ }^{\mathrm{g}}$ AMC, Academic Psychiatric Centre, Department Early Psychosis, Amsterdam, the Netherlands

${ }^{\mathrm{h}}$ Arkin, Amsterdam, the Netherlands

${ }^{\mathrm{i}}$ Centre for Youth Mental Health, University of Melbourne, Melbourne, Australia

j Departament de Psicologia Clínica I de la Salut (Universitat Autònoma de Barcelona), Fundació Sanitària Sant Pere Claver (Spain), Spanish Mental Health Research Network (CIBERSAM), Spain

${ }^{\mathrm{k}}$ Mental Health Center Copenhagen, Mental Health Services in the Capital Region of Denmark, Denmark

${ }^{1}$ Institute for Clinical Medicine, Faculty of Health Science, University of Copenhagen, Denmark

m Department of Psychiatry and Psychotherapy, University of Cologne, Cologne, Germany

${ }^{\mathrm{n}}$ Medical University of Vienna, Department of Psychiatry and Psychotherapy, Austria

${ }^{\circ}$ Department of Psychiatry and Neuropsychology, School for Mental Health and Neuroscience, Maastricht, the Netherlands

P Department Psychiatry, Brain Centre Rudolf Magnus, Utrecht University Medical Centre, Utrecht, the Netherlands

${ }^{\mathrm{q}}$ Department of Psychiatry and Psychology, School for Mental Health and Neuroscience (MHeNS), Maastricht University Medical Centre, Maastricht, the Netherlands

${ }^{\mathrm{r}}$ King's College London, King's Health Partners Department of Psychosis Studies, Institute of Psychiatry, London, United Kingdom

\section{A R T I C L E I N F O}

\section{Article history:}

Received 13 March 2019

Received in revised form 25 April 2019

Accepted 26 April 2019

Available online 7 May 2019

\section{Keywords:}

Gender differences

Sex differences

Risk for psychosis

Comorbidity

Functioning

\begin{abstract}
A B S T R A C T
Background: Gender differences in symptomatology in chronic schizophrenia and first episode psychosis patients have often been reported. However, little is known about gender differences in those at risk of psychotic disorders. This study investigated gender differences in symptomatology, drug use, comorbidity (i.e. substance use, affective and anxiety disorders) and global functioning in patients with an at-risk mental state (ARMS) for psychosis.

Methods: The sample consisted of 336 ARMS patients (159 women) from the prodromal work package of the EUropean network of national schizophrenia networks studying Gene-Environment Interactions (EU-GEI; 11 centers). Clinical symptoms, drug use, comorbidity and functioning were assessed at first presentation to an early detection center using structured interviews.

Results: In unadjusted analyses, men were found to have significantly higher rates of negative symptoms and current cannabis use while women showed higher rates of general psychopathology and more often displayed comorbid affective and anxiety disorders. No gender differences were found for global functioning. The results generally did not change when corrected for possible cofounders (e.g. cannabis
\end{abstract}

\footnotetext{
* Corresponding author at: University of Basel Psychiatric Hospital, Center for Gender Research and Early Detection, Wilhelm Klein-Strasse 27, 4002 Basel, Switzerland. E-mail address: anita.riecher@upk.ch (A. Riecher-Rössler).

1 A full list of authors and affiliations appears in the Appendix.
} 
use). However, most differences did not withstand correction for multiple testing.

Conclusions: Findings indicate that gender differences in symptomatology and comorbidity in ARMS are similar to those seen in overt psychosis and in healthy controls. However, observed differences are small and would only be reliably detected in studies with high statistical power. Moreover, such small effects would likely not be clinically meaningful.

(c) 2019 Published by Elsevier Masson SAS.

\section{Introduction}

Gender differences in schizophrenia have attracted the attention of scientific research for more than a century. Kraepelin had already reported that women are older at first admission for dementia praecox compared to men [1]. Most studies to date confirm these findings [2]. Findings on severity of psychopathological symptoms are less conclusive, with some authors suggesting that men have more severe negative symptoms while women show more severe affective and specific psychotic symptoms [2]. However, only few gender differences in psychopathology of first episode schizophrenia were found in the ABC study, and these were not significant after correction for multiple testing $[3,4]$. With regard to substance abuse, available evidence suggests that men have a higher prevalence of substance abuse and higher levels of comorbidity compared to women. Additionally, studies examining gender differences in premorbid and social functioning have found higher functioning in women [2].

In the past two decades, the field of early detection of psychosis has received growing scientific and clinical interest [5], albeit that only few methodologically sound studies have considered gender differences in patients with an at-risk mental state (ARMS) for psychosis. These studies have thus far yielded inconsistent results. With regard to symptomatology, most studies described in the comprehensive review of Barajas et al. [6] reported no gender differences in ARMS patients. Nevertheless, some studies found more severe negative symptoms in men, and other studies found lower levels of social functioning and a longer duration of untreated illness in men compared to women [6]. A more recent review published by Riecher-Rössler et al. [2] suggests that gender differences in the symptomatology of patients at risk are small and comparable to those seen in the general population. Thus, in a representative worldwide general population sample of 72,933 subjects, men in general had a greater propensity to substance, alcohol and cannabis abuse, while women had more affective symptoms, depression and anxiety [7].

In addition to the at-risk signs and symptoms for psychosis, many ARMS patients suffer from comorbid non-psychotic mental disorders, in particular depression and anxiety disorders [8,9]. To our knowledge, only two studies have investigated gender differences in comorbid depressive and anxiety diagnoses in ARMS patients at baseline. Kline et al. [10] examined a cohort of 764 ARMS patients (women, $\mathrm{n}=329 ; 43 \%$ ) from the North American Prodrome Longitudinal Study $\left(\mathrm{NAPLS}^{-2}\right.$ ), and observed a significantly higher lifetime prevalence of depression in women than men (64\% vs. 56\%). No significant gender differences in comorbid affective and anxiety disorders were observed in the study of Rietschel et al. [11].

To further elucidate these issues, the present study investigated gender differences in symptomatology, drug use, comorbidity (i.e. substance use, affective and anxiety disorders) and global functioning in a large multinational sample of ARMS patients. Based on previous and our own findings, we expected to find no significant differences between ARMS men and women.

\section{Methods}

\subsection{Setting and recruitment}

The data analysed in this study were collected within the multicenter EUropean Gene-Environment Interactions (EU-GEI) study, from May 1, 2010 to April 30, 2015. The aim of EU-GEI study is to identify the interactive genetic, clinical and environmental determinants of schizophrenia [12]. The overall design of the study was naturalistic, longitudinal and prospective, consisting of a baseline and two follow-up time points. For the current analyses, only baseline, i.e. at intake into the study, data were used and only patients with complete data on cannabis frequency were included.

ARMS patients were recruited from 11 Early Detection and Intervention Centers, nine in Europe (London, Amsterdam, The Hague, Vienna, Basel, Cologne, Copenhagen, Paris, Barcelona), one in Brazil (Saõ Paulo), and one in Australia (Melbourne). Referrals were accepted from primary health care services, mental health professionals, or from the subject or their family. Study intake corresponds to the admission date in the early detection service. All participants were screened with an inclusion/exclusion checklist (see below).

The protocol of the EU-GEI study was approved by the institutional review boards of all study sites. EU-GEI was conducted in accordance with the Declaration of Helsinki. The Medical Ethics Committees of all participating sites approved the study protocol.

\subsection{Inclusion and exclusion criteria}

Inclusion criteria for EU-GEI were: aged 18-35; being at-risk for psychosis as defined by the Comprehensive Assessment of At-Risk Mental State (CAARMS) [13]; adequate language skills local to each center; and consent to study participation.

The exclusion criteria were: prior experience of a psychotic episode of more than 1-week as determined by the CAARMS [13] and Structural Clinical Interview for DSM Disorders (SCID) [14]; previous treatment with an antipsychotic for a psychotic episode; and IQ $<60$.

\subsection{Determination of ARMS status}

The CAARMS, used to identify ARMS patients [13], is a semistructured interview that encompasses psychotic symptoms and a range of other psychopathological symptoms occurring in emerging psychotic disorder. Individuals were classified as being in an ARMS for psychosis if they met at least one of the following risk criteria: (i) Vulnerability Group (a first-degree relative with a psychotic disorder or diagnosed with schizotypal personality disorder in combination with a significant drop in functioning); (ii) Attenuated Psychotic Symptoms (APS) (psychotic symptoms sub-threshold either in intensity or frequency); (iii) Brief Limited Psychotic Symptoms (BLIPS) (recent episode of brief psychotic symptoms that spontaneously resolved within 1 week). The full criteria can be found in Yung et al. [13]. 


\subsection{Assessment of sociodemographic characteristics and medication}

Sociodemographic characteristics were obtained using the modified Medical Research Council (MRC) sociodemographic schedule [15]. Data on psychiatric medication were assessed with a medical history questionnaire, designed by the EU-GEI group.

\subsection{Assessment of psychopathology}

Psychopathological symptoms were assessed using the expanded version of the Brief Psychiatric Rating Scale (BPRS-E) [16], the Scale for the Assessment of Negative Symptoms (SANS) [17], the Comprehensive Assessment of At-Risk Metal State (CAARMS) [13], the Montgomery-Åsberg Depression Rating Scale (MADRS) [18], and the Young Mania Rating Scale (YMRS) [19]. Genders differences were investigated using the following subscales:

BPRS-E: Activation, Positive symptoms, Negative symptoms, Affect, Disorganization as defined by Shafer et al. [16] and the total score

SANS: Affective Flattening, Alogia, Asociality-Anhedonia, Avolition-Apathy, Inattention and the total score [17]

CAARMS: Behavioral change, Cognitive change - attention/ concentration, Emotional disturbance, Motor/physical changes, Negative symptoms, Positive symptoms, General Psychopathology[20]

MADRS: Detachment, Negative Thoughts, Neurovegetative, Sadness as defined by Quilty et al. [21] and the total score

YMRS: Total score [19]

\subsection{Assessment of comorbidity, drug use and functioning}

Affective and anxiety disorders were assessed with the Structured Clinical Interview for the Diagnostic Manual of Psychiatric Disorders-IV (DSM-IV/SCID) [14]. Current use, abuse and dependence of cannabis, amphetamine (e.g. speed, ecstasy), cocaine, and hallucinogens (e.g. lysergic acid diethylamide (LSD), "magic mushrooms") were assessed using the Cannabis Experience Questionnaire [22]. For cannabis, the frequency of use was additionally assessed. Participants were defined as being current users of a substance if they identified themselves as such or if they reported any use in the preceding month.

The general level of functioning was assessed with the GAF scale [23].

\subsection{Statistical analyses}

All statistical analyses were carried out using R environment for statistical computing [24]. Because observations were nonindependent, that is, observations from the same center were more similar than observations from different centers, gender differences were analysed using mixed effects models including gender as a fixed effects factor and randomly varying intercepts per center to account for the clustering in the data. We used linear mixed effects models for continuous measures (i.e. age, years of education, functioning and psychopathology scales), mixed effects logistic regression models for binary measures (i.e. psychiatric diagnoses, drug use and psychiatric medication), ordinal mixed effects models for ordered categorical measures (i.e. cannabis frequency and highest level of education) and mixed effects multinomial logistic regression for unordered categorical measures (i.e. living situation). We analysed gender differences in the frequency of use of antipsychotics, antidepressants and hypnotics. Cannabis frequency and age were included as covariates in models estimating gender differences in psychopathology and living situation, respectively. Continuous dependent variables were z-transformed before inclusion to models and gender was included as a binary variable with 0 and 1 describing men and women, respectively. Thus, the regression coefficient for gender described the standardized mean difference of women compared to men. $P$-values were adjusted for multiple testing across all of the 63 gender differences tests using the False Discovery Rate (FDR) procedure [25].

\section{Results}

\subsection{Sample description}

In total, 345 ARMS patients participated in the EU-GEI study. The sample of this study consisted of 336 ARMS patients (177 men, 159 women). 9 ARMS patients had not complete data on cannabis frequency and were excluded. Sociodemographic and clinical characteristics are presented in Table 1. Male patients were significantly older than female patients in unadjusted analyses $(P=0.011)$. The significance of this effect disappeared after correction for multiple testing $(P=0.175)$. There were no significant gender differences in ARMS patients with regard to years of education, highest level of education, living situation and current psychiatric medication.

Table 1

Sociodemographic and clinical characteristics.

\begin{tabular}{|c|c|c|c|c|c|}
\hline & Men $(n=177)$ & Women $(n=159)$ & $\mathrm{N}$ & $P$-value uncorrected & $P$-value corrected ${ }^{\mathrm{a}}$ \\
\hline Age & $22.8(5.13)$ & $22.0(4.70)$ & 336 & $0.011^{*}$ & 0.175 \\
\hline Years of education & $14.4(3.29)$ & $14.4(2.84)$ & 301 & 0.190 & 0.471 \\
\hline Highest level of education & & & 296 & 0.987 & 1.000 \\
\hline School, no qualifications & $16(9.88 \%)$ & $11(8.21 \%)$ & & & \\
\hline School, with qualifications & $51(31.5 \%)$ & $47(35.1 \%)$ & & & \\
\hline Tertiary, Further & $50(30.9 \%)$ & $38(28.4 \%)$ & & & \\
\hline Vocational & $24(14.8 \%)$ & $17(12.7 \%)$ & & & \\
\hline Higher (undergraduate) & $18(11.1 \%)$ & $17(12.7 \%)$ & & & \\
\hline Higher (postgraduate) & $3(1.85 \%)$ & $4(2.99 \%)$ & & & \\
\hline Living with & & & 336 & 0.575 & $0.471^{\mathrm{b}}$ \\
\hline Alone & $28(15.8 \%)$ & $23(14.5 \%)$ & & & \\
\hline Other & $56(31.6 \%)$ & $61(38.4 \%)$ & & & \\
\hline Parents/family & $93(52.5 \%)$ & $75(47.2 \%)$ & & & \\
\hline Antipsychotics currently & $15(10.3 \%)$ & $15(11.3 \%)$ & 279 & 0.911 & 0.988 \\
\hline Antidepressants currently & $41(28.1 \%)$ & $43(32.3 \%)$ & 279 & 0.909 & 0.988 \\
\hline Hypnotics currently & $2(1.37 \%)$ & $2(1.50 \%)$ & 279 & 0.994 & 1.000 \\
\hline
\end{tabular}

Continuous variables are described by means and standard deviations in parentheses.

a $P$-value corrected for multiple testing.

b $P$-value corrected for age and multiple testing.

$P<0.05$. 


\subsection{Gender differences in symptomatology and functioning}

Table 2 shows the results of the linear mixed effects models using symptomatology as continuous dependent variable and gender as fixed effects factor. Standardized mean differences (SMD) and 95\% confidence intervals of the psychopathological syndrome scales are additionally presented in Fig. 1.

Female ARMS patients showed significantly less severe BPRS "Negative Symptoms" $(b=-0.22, P=0.046)$, more CAARMS "General psychopathology" ( $b=0.30, P=0.007)$ and trendwise less SANS "Affective Flattening" ( $b=-0.20, P=0.073$ ) than male ARMS patients in uncorrected analyses. These differences became significant when corrected for cannabis use (BPRS: $b=-0.24$, $P=0.032$; CAARMS: $b=0.33, P=0.003$, SANS: $b=-0.22, P=0.048$ ). However, when $p$-values were additionally adjusted for multiple testing by using the FDR procedure, differences in negative symptoms and general psychopathology were no longer significant. There were no gender differences in ARMS patients with regard to global functioning.

\subsection{Gender differences in drug use and comorbidity}

Table 3 shows the ORs for associations of gender with comorbid drug use and affective and anxiety disorders for ARMS patients at

Table 2

Gender differences in psychopathology and functioning.

\begin{tabular}{|c|c|c|c|c|c|c|c|c|}
\hline Rating scale & $\begin{array}{l}\text { Men } \\
(n=177)\end{array}$ & $\begin{array}{l}\text { Women } \\
(\mathrm{n}=159)\end{array}$ & $\mathrm{N}$ & $\begin{array}{l}\text { Coefficient }[\mathrm{CI}] \\
\text { uncorrected }\end{array}$ & $\begin{array}{l}\text { Coefficient }[\mathrm{CI}] \text { corrected } \\
\text { cannabis use }\end{array}$ & $\begin{array}{l}P \text {-value } \\
\text { uncorrected }\end{array}$ & $\begin{array}{l}P \text {-value corrected for } \\
\text { cannabis use }\end{array}$ & $\begin{array}{l}P \text {-value fully } \\
\text { corrected }^{\mathrm{a}}\end{array}$ \\
\hline \multicolumn{9}{|l|}{ BPRS } \\
\hline BPRS Activation & $3.8(1.7)$ & $3.5(1.3)$ & 319 & $\begin{array}{l}-0.15[-0.37 \\
0.07]\end{array}$ & $-0.10[-0.32 ; 0.12]$ & 0.191 & 0.396 & 0.700 \\
\hline BPRS Affect & $7.7(3.2)$ & $8.1(3.1)$ & 319 & $0.02[-0.20 ; 0.25]$ & $0.06[-0.16 ; 0.29]$ & 0.848 & 0.613 & 0.866 \\
\hline BPRS Disorganization & $4.0(1.5)$ & $3.6(1.3)$ & 319 & $\begin{array}{l}-0.13[-0.35 \\
0.09]\end{array}$ & $-0.09[-0.32 ; 0.13]$ & 0.241 & 0.412 & 0.700 \\
\hline BPRS Negative Symptoms & $5.4(2.7)$ & $4.7(2.0)$ & 319 & $\begin{array}{l}-0.22[-0.44 \\
-0.01]\end{array}$ & $-0.24[-0.46 ;-0.02]$ & $0.046^{*}$ & $0.032^{*}$ & 0.181 \\
\hline BPRS Positive Symptoms & $7.7(3.3)$ & $7.3(3.0)$ & 318 & $\begin{array}{l}-0.09[-0.31 \\
0.12]\end{array}$ & $-0.05[-0.27 ; 0.17]$ & 0.406 & 0.663 & 0.886 \\
\hline BPRS total score & $\begin{array}{l}44.2 \\
(10.8)\end{array}$ & $43.0(9.7)$ & 319 & $\begin{array}{l}-0.07[-0.29 \\
0.14]\end{array}$ & $-0.03[-0.24 ; 0.19]$ & 0.498 & 0.798 & 0.928 \\
\hline \multicolumn{9}{|l|}{ CAARMS } \\
\hline CAARMS Behavioral change & $7.8(4.2)$ & $8.2(3.7)$ & 332 & $0.07[-0.15 ; 0.30]$ & $0.11[-0.11 ; 0.33]$ & 0.520 & 0.336 & 0.700 \\
\hline $\begin{array}{l}\text { CAARMS Cognitive change, } \\
\text { attention/concentration }\end{array}$ & $3.2(1.8)$ & $3.1(1.8)$ & 332 & $0.03[-0.20 ; 0.25]$ & $0.04[-0.19 ; 0.26]$ & 0.804 & 0.740 & 0.925 \\
\hline CAARMS Emotional disturbance & $3.2(2.3)$ & $3.1(2.5)$ & 330 & $\begin{array}{l}-0.02[-0.24 \\
0.20]\end{array}$ & $-0.04[-0.26 ; 0.18]$ & 0.862 & 0.751 & 0.925 \\
\hline $\begin{array}{l}\text { CAARMS General } \\
\text { psychopathology }\end{array}$ & $\begin{array}{l}13.8 \\
(6.6)\end{array}$ & $16.1(6.0)$ & 333 & $0.30[0.08 ; 0.52]$ & $0.33[0.12 ; 0.56]$ & $0.007^{* *}$ & $0.003^{* *}$ & 0.087 \\
\hline $\begin{array}{l}\text { CAARMS Motor/physical } \\
\text { changes }\end{array}$ & $2.1(2.7)$ & $2.3(2.5)$ & 329 & $0.18[-0.04 ; 0.40]$ & $0.19[-0.03 ; 0.41]$ & 0.107 & 0.088 & 0.312 \\
\hline CAARMS Negative symptoms & $6.7(3.7)$ & $7.2(3.1)$ & 331 & $0.10[-0.11 ; 0.34]$ & $0.12[-0.10 ; 0.35]$ & 0.347 & 0.298 & 0.700 \\
\hline CAARMS Positive symptoms & $\begin{array}{l}10.0 \\
(3.9)\end{array}$ & $9.7(4.4)$ & 334 & $0.00[-0.21 ; 0.21]$ & $0.02[-0.20 ; 0.23]$ & 0.993 & 0.862 & 0.985 \\
\hline \multicolumn{9}{|l|}{ GAF } \\
\hline GAF Disability, impairment & $\begin{array}{l}55.6 \\
(12.4)\end{array}$ & $55.2(12.4)$ & 328 & $0.05[-0.18 ; 0.26]$ & $0.01[-0.22 ; 0.22]$ & 0.682 & 0.960 & 1.000 \\
\hline GAF Symptoms & $\begin{array}{l}54.9 \\
(10.3)\end{array}$ & $55.3(10.1)$ & 313 & $0.07[-0.16 ; 0.29]$ & $0.05[-0.18 ; 0.28]$ & 0.570 & 0.678 & 0.886 \\
\hline \multicolumn{9}{|l|}{ MADRS } \\
\hline MADRS Detachment & $6.4(3.2)$ & $6.2(3.2)$ & 323 & $\begin{array}{l}-0.07[-0.29 \\
0.15]\end{array}$ & $-0.07[-0.29 ; 0.16]$ & 0.546 & 0.568 & 0.846 \\
\hline MADRS Negative Thoughts & $3.0(2.3)$ & $3.2(2.1)$ & 322 & $0.07[-0.15 ; 0.31]$ & $0.10[-0.12 ; 0.35]$ & 0.528 & 0.374 & 0.700 \\
\hline MADRS Neurovegetative & $5.0(3.1)$ & $5.4(3.3)$ & 323 & $0.14[-0.09 ; 0.36]$ & $0.17[-0.06 ; 0.39]$ & 0.228 & 0.147 & 0.410 \\
\hline MADRS Sadness & $4.2(2.7)$ & $4.4(2.8)$ & 323 & $0.03[-0.19 ; 0.26]$ & $0.03[-0.19 ; 0.26]$ & 0.759 & 0.773 & 0.927 \\
\hline MADRS total score & $\begin{array}{l}18.6 \\
(9.3)\end{array}$ & $19.2(9.1)$ & 323 & $0.06[-0.16 ; 0.28]$ & $0.08[-0.14 ; 0.31]$ & 0.581 & 0.464 & 0.724 \\
\hline \multicolumn{9}{|l|}{ SANS } \\
\hline SANS Affective Flattening & $4.0(4.7)$ & $2.9(4.2)$ & 325 & $\begin{array}{l}-0.20[-0.42 \\
0.02]\end{array}$ & $-0.22[-0.45 ; 0.00]$ & 0.073 & $0.048^{*}$ & 0.238 \\
\hline SANS Alogia & $1.8(2.5)$ & $1.0(2.1)$ & 325 & $\begin{array}{l}-0.17[-0.38 \\
0.04]\end{array}$ & $-0.17[-0.39 ; 0.04]$ & 0.120 & 0.121 & 0.381 \\
\hline SANS Asociality-Anhedonia & $6.1(4.6)$ & $5.1(4.0)$ & 324 & $\begin{array}{l}-0.16[-0.39 \\
0.06]\end{array}$ & $-0.18[-0.41 ; 0.05]$ & 0.157 & 0.125 & 0.381 \\
\hline SANS Avolition-Apathy & $3.5(2.9)$ & $3.4(2.6)$ & 325 & $0.06[-0.15 ; 0.27]$ & $0.08[-0.13 ; 0.30]$ & 0.576 & 0.449 & 0.724 \\
\hline SANS Inattention & $0.9(1.5)$ & $1.1(1.7)$ & 321 & $0.20[-0.02 ; 0.41]$ & $0.20[-0.02 ; 0.41]$ & 0.069 & 0.079 & 0.312 \\
\hline SANS total score & $\begin{array}{l}16.4 \\
(11.5)\end{array}$ & $13.5(10.8)$ & 325 & $\begin{array}{l}-0.14[-0.35 \\
0.08]\end{array}$ & $-0.15[-0.37 ; 0.07]$ & 0.216 & 0.191 & 0.471 \\
\hline \multicolumn{9}{|l|}{ YMRS } \\
\hline YMRS total score & $4.4(5.1)$ & $3.3(3.8)$ & 316 & $\begin{array}{l}-0.17[-0.38 \\
0.05]\end{array}$ & $-0.09[-0.30 ; 0.12]$ & 0.131 & 0.415 & 0.700 \\
\hline
\end{tabular}

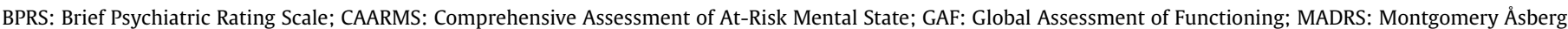
Depression Rating Scale;

SANS: Scale for the Assessment of Negative Symptoms; YMRS: Young Mania Rating Scale; $\mathrm{CI}=95 \%$ Confidence Interval .

${ }^{a} P$-value corrected for cannabis use and multiple testing.

* $P<0.05$.

${ }^{* *} P<0.01$ 


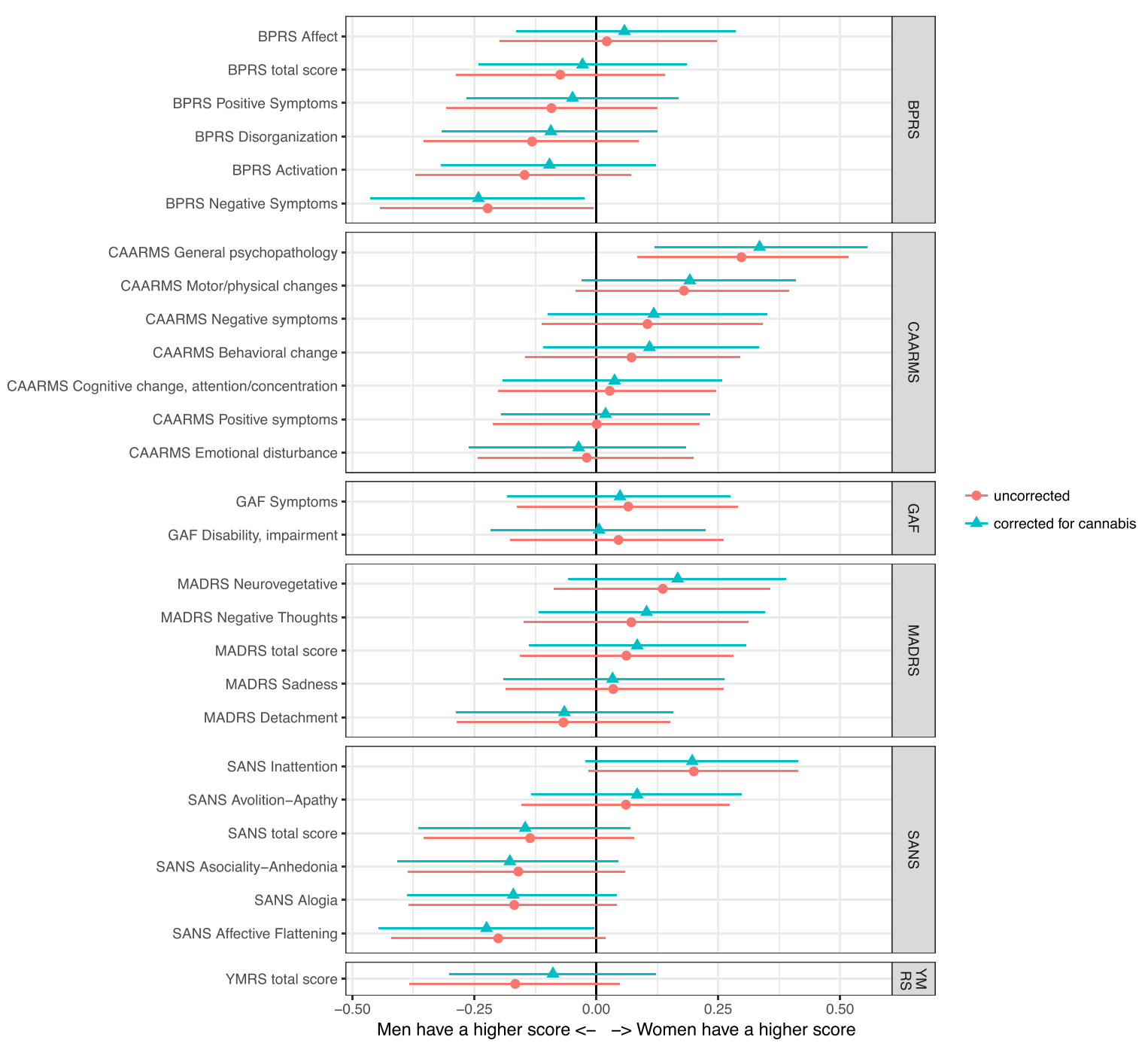

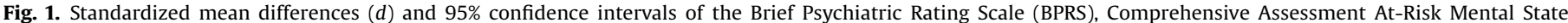

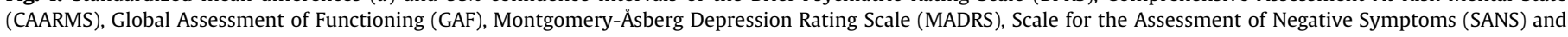

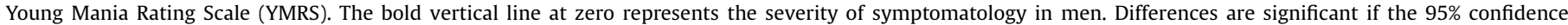
interval (horizontal line) does not overlap with zero.

baseline. Unadjusted ORs indicate that men had a significantly higher proportion of current cannabis users (OR, 0.53; 95\% CI 0.32 to $0.88 ; P=0.015$ ) and a higher current frequency of cannabis use than women $(P=0.008)$.

With regard to broad diagnostic categories, women were significantly more often diagnosed with any lifetime affective disorder (OR, 1.72; 95\% CI 1.05-2.81; $P=0.032$ ) and any current anxiety disorder (OR, 1.66; 95\% CI 1.04-2.64; $P=0.034$ ). With regard to specific diagnoses, women were more frequently diagnosed with a past major depressive episode (OR, 1.78; 95\% CI 1.11-2.88; $P=0.018)$, a current panic disorder with $(\mathrm{OR}, 2.57 ; 95 \%$ CI 1.14-5.81; $P=0.024)$ and without agoraphobia (OR, 2.00; 95\% CI $1.12-3.55 ; P=0.019$ ), current specific phobia (OR, 4.26; $95 \%$ CI 1.90-9.51; $P=<0.001$ ) and current PTSD (OR, 2.25; 95\% CI 1.07-4.74; $P=0.033$ ). However, when adjusted for multiple testing, only current specific phobia remained significantly associated with gender $(P=0.031)$.

\section{Discussion}

The current study investigated gender differences in sociodemographic variables, symptomatology, drug use, comorbidity (i.e. substance use, affective and anxiety disorders) and global functioning in 336 ARMS patients presenting for the first time at an early detection service in a multi-national study. Unadjusted analyses indicated higher severity of negative symptoms (i.e. BPRS negative symptoms, SANS affective flattening) and current cannabis use in men while women showed higher severity of general psychopathology (CAARMS) and suffered more from comorbid affective (i.e. lifetime affective disorders, past major depressive episode) and anxiety disorders (e.g. panic, panic with agoraphobia, specific phobia, PTSD). However, when corrected for multiple testing and confounding variables, these differences were no longer significant except for higher lifetime rates of specific phobia in women.

Regarding sociodemographic variables, our results are in agreement with an earlier study on ARMS patients [11] with the exception of age and living situation. While Rietschel et al. [11] found no gender difference in age, the current study found male ARMS patients to be significantly older than female ARMS patients but only if statistically not corrected for multiple testing. Rietschel et al. [11] suggest that male ARMS patients are living more frequently with their parents or other relatives than female ARMS patients whereas the present study did not find any significant 
Table 3

Gender differences in drug use and comorbidity.

\begin{tabular}{|c|c|c|c|c|c|c|c|}
\hline SCID Diagnosis & \multicolumn{2}{|c|}{ Men $(n=177)$} & Women $(n=159)$ & $\mathrm{N}$ & Odds ratio $[\mathrm{CI}]$ & $P$-value uncorrected & $P$-value corrected ${ }^{\mathrm{a}}$ \\
\hline \multicolumn{8}{|l|}{ Drug use } \\
\hline Cannabis current use & \multicolumn{2}{|l|}{$56(31.6 \%)$} & $31(19.5 \%)$ & 336 & $0.53[0.32 ; 0.88]$ & $0.015^{*}$ & 0.175 \\
\hline Cannabis current frequency & & & & 336 & & $0.008^{* * *}$ & 0.168 \\
\hline none & \multicolumn{2}{|l|}{$121(68.4 \%)$} & $128(80.5 \%)$ & & & & \\
\hline only once or twice & \multicolumn{2}{|l|}{$2(1.13 \%)$} & $2(1.26 \%)$ & & & & \\
\hline a few times each year & \multicolumn{2}{|l|}{7 (3.95\%) } & $10(6.29 \%)$ & & & & \\
\hline a few times each month & \multicolumn{2}{|l|}{$12(6.78 \%)$} & $5(3.14 \%)$ & & & & \\
\hline (more than) once a week & \multicolumn{2}{|l|}{$9(5.08 \%)$} & $2(1.26 \%)$ & & & & \\
\hline every day & \multicolumn{2}{|l|}{$26(14.7 \%)$} & $12(7.55 \%)$ & & & & \\
\hline Cannabis lifetime dependence & \multicolumn{2}{|l|}{$36(35.6 \%)$} & $21(26.9 \%)$ & 179 & $0.72[0.38 ; 1.40]$ & 0.337 & 0.714 \\
\hline Amphetamines current use & \multicolumn{2}{|l|}{$30(26.1 \%)$} & $17(21.0 \%)$ & 196 & $0.74[0.37 ; 1.46]$ & 0.383 & 0.721 \\
\hline Amphetamines current abuse & \multicolumn{2}{|l|}{$2(1.7 \%)$} & $2(2.5 \%)$ & 196 & $1.43[0.20 ; 10.48]$ & 0.725 & 0.910 \\
\hline Amphetamines current dependence & \multicolumn{2}{|l|}{$2(1.7 \%)$} & $3(3.7 \%)$ & 196 & $2.23[0.42 ; 11.97]$ & 0.351 & 0.714 \\
\hline Cocaine current use & \multicolumn{2}{|l|}{$28(24.3 \%)$} & $11(13.6 \%)$ & 196 & $0.49[0.23 ; 1.05]$ & 0.069 & 0.312 \\
\hline Cocaine current abuse & \multicolumn{2}{|l|}{$2(1.7 \%)$} & $2(2.5 \%)$ & 196 & $1.52[0.39 ; 5.84]$ & 0.544 & 0.808 \\
\hline Cocaine current dependence & \multicolumn{2}{|l|}{$2(1.7 \%)$} & $1(1.2 \%)$ & 196 & $0.69[0.16 ; 2.98]$ & 0.619 & 0.829 \\
\hline Hallucinogens current use & \multicolumn{2}{|l|}{$16(13.9 \%)$} & $9(11.1 \%)$ & 196 & $0.81[0.34 ; 1.89]$ & 0.622 & 0.829 \\
\hline Hallucinogens current abuse & \multicolumn{2}{|l|}{$1(0.9 \%)$} & $1(1.2 \%)$ & 196 & $1.71[0.42 ; 6.96]$ & 0.456 & 0.788 \\
\hline Hallucinogens current dependence & \multicolumn{2}{|l|}{$1(0.9 \%)$} & $0(0.0 \%)$ & 196 & $0.00[0.00 ; 0.00]$ & 1.000 & 1.000 \\
\hline \multicolumn{8}{|l|}{ Affective disorders } \\
\hline Lifetime affective disorder & \multicolumn{2}{|l|}{$106(59.9 \%)$} & $122(76.7 \%)$ & 336 & $1.72[1.05 ; 2.81]$ & $0.032^{*}$ & 0.198 \\
\hline Current major depressive episode & \multicolumn{2}{|l|}{$48(27.6 \%)$} & $59(38.6 \%)$ & 327 & $1.52[0.93 ; 2.46]$ & 0.093 & 0.329 \\
\hline Past major depressive episode & \multicolumn{2}{|l|}{$71(41.8 \%)$} & $84(57.5 \%)$ & 316 & $1.78[1.11 ; 2.88]$ & $0.018^{*}$ & 0.175 \\
\hline Current dysthymic disorder & \multicolumn{2}{|l|}{$10(6.0 \%)$} & $10(6.7 \%)$ & 317 & $0.89[0.37 ; 2.10]$ & 0.782 & 0.945 \\
\hline Past manic episode & \multicolumn{2}{|l|}{$5(3.0 \%)$} & $7(4.7 \%)$ & 319 & $1.64[0.54 ; 5.00]$ & 0.385 & 0.721 \\
\hline Current hypomaniac episode & \multicolumn{2}{|l|}{$3(1.9 \%)$} & $1(0.7 \%)$ & 306 & $0.37[0.04 ; 3.63]$ & 0.395 & 0.721 \\
\hline Past hypomaniac episode & $8(4.8 \%)$ & & $9(6.1 \%)$ & 314 & $1.24[0.47 ; 3.26]$ & 0.665 & 0.868 \\
\hline Anxiety disorders & & & & & & & \\
\hline Current anxiety disorder & & $71(40.1 \%)$ & $94(59.1 \%)$ & 336 & $1.66[1.04 ; 2.64]$ & $0.034^{*}$ & 0.198 \\
\hline Current panic disorder & & $26(15.7 \%)$ & $44(29.5 \%)$ & 315 & $2.00[1.12 ; 3.55]$ & $0.019^{*}$ & 0.175 \\
\hline Current panic disorder with agora & & $9(5.9 \%)$ & $21(15.3 \%)$ & 290 & $2.57[1.14 ; 5.81]$ & $0.024^{*}$ & 0.194 \\
\hline Current agoraphobia without histo & c disorder & $3(1.7 \%)$ & $3(1.9 \%)$ & 336 & $0.90[0.18 ; 4.53]$ & 0.895 & 0.988 \\
\hline Current social phobia & & $23(13.6 \%)$ & $38(25.5 \%)$ & 318 & $1.66[0.92 ; 3.01]$ & 0.092 & 0.329 \\
\hline Current specific phobia & & $7(4.2 \%)$ & $29(19.3 \%)$ & 318 & $4.26[1.90 ; 9.51]$ & $<0.001^{* * *}$ & $0.031^{*}$ \\
\hline Current generalized anxiety disord & & $14(8.5 \%)$ & $22(14.6 \%)$ & 315 & $1.69[0.84 ; 3.40]$ & 0.144 & 0.419 \\
\hline Current obsessive compulsive diso & & $16(10.3 \%)$ & $13(9.3 \%)$ & 295 & $0.99[0.46 ; 2.13]$ & 0.987 & 1.000 \\
\hline Current post traumatic stress disor & & $11(6.6 \%)$ & $23(15.3 \%)$ & 317 & $2.25[1.07 ; 4.74]$ & $0.033^{*}$ & 0.198 \\
\hline
\end{tabular}

SCID: Structured Clinical Interview for the Diagnostic Manual of Psychiatric Disorders DSM-IV; CI: 95\% Confidence Interval.

${ }^{a} P$-value corrected for multiple testing.

${ }^{*} P<0.05$

${ }^{* *} P<0.01$

*** $P<0.001$

gender differences. This finding may be due to the slightly lower average age in our sample. Another possibility is that this gender difference is dependent on the country or region the sample is taken from.

Regarding psychopathology, our findings were in line with a previous study of our own group that reported no gender differences in psychopathology, neither in ARMS nor in FEP patients, when corrected for multiple testing [26]. Furthermore, Willhite et al. [27] also found no significant gender differences in ratings of any of the symptoms of the Scale of Prodromal Symptoms (SOPS) in high-risk patients. A possible explanation may be that gender differences in the symptoms are so small that they can only be reliably detected in studies with very high statistical power (i.e. in very large datasets or in meta-analyses). However, such small effects would likely not be clinically meaningful.

Regarding drug use and comorbidity, male ARMS patients showed higher rates of current cannabis use and frequency of intake in unadjusted but not in adjusted analyses compared to female ARMS patients. This finding is in line with a previous study of our own group [26] and others that report no gender differences regarding substance abuse in the prodromal phase of schizophrenia [2]. However, higher rates of substance abuse in men are found in the general population [7] and in schizophrenia in particular [2].
Our finding of higher rates of comorbid affective and anxiety disorders in female ARMS patients contradicts a recent study on ARMS patients, which has found no gender differences for affective and anxiety disorders [11]. However, an earlier study found greater rates of current depression and social anxiety in high-risk women compared to men [28]. Furthermore, Pruessner et al. [29] also found more depressive symptoms in high-risk women, but these differences did not withstand correction for multiple testing. An explanation may be that the self-report questionnaires used in the study of Rietdijk et al. [28] have led to an overestimation of the number of patients with an anxiety disorder or depression. Most importantly, our results are in line with epidemiological studies on depression and anxiety in the general population, which found female/male prevalence ratios of 2:1, respectively [30,31]. ARMS patients in this respect thus do not seem to differ from the general population.

Our finding of no gender difference in terms of level of functioning is in accordance with previous studies [2].

A strength of our study is that we examined gender differences with several, well established instruments to assess a broad range of symptomatology. Rater trainings have been used to ensure that all raters administering the rating scales in the same way. Furthermore, the multicentre design of our study might have contributed to heterogeneity in our sample through, for example, 
different cultural modes of expression and accessibility and potency of cannabis products in different study centres. We have therefore included random intercepts that varied across study centres in all our models. Finally, this is one of the first studies to investigate gender differences in symptomatology in an ARMS sample of this size.

However, although data were collected by well-trained interviewers using standardized questionnaires and wellestablished diagnostic criteria, this does not completely eliminate possible gender-specific biases, e.g. of questionnaires and interviewing techniques, of self-reporting, or interpreting patient information, of applying diagnostic criteria or attributing diagnostic labels [32]. Furthermore, this study concentrates on the age group of 18-35 years with the consequence that especially boys, who are at-risk state presumably before age 18 and women with later age of onset are missed. An additional limitation could be that our sample may not be representative for the overall population of help-seeking patients since we do not know whether all ARMS patients in the relevant catchment areas were searching help and came to an early detection service. A recent study found a significantly different gender distribution between ARMS and first episode psychosis (FEP) patients with a greater proportion of males in FEP cohorts than in clinical high-risk cohorts [33]. The authors presume that ARMS men are probably less likely to be help-seeking or less 'literate' of symptoms of mental illness which could lead to an under-representation of men in existing clinical high-risk services. Lastly, it should be noted that ARMS patients represent a heterogeneous patient group with only about 20-35\% developing frank psychosis [34,35] and about one third having a clinical remission within the first two years of the follow-up [36]. Hence, gender differences reported in this study cannot be generalized to patients being in true prodromal state for psychosis.

Taken together, our findings indicate that gender differences in symptomatology - if present at all - are so small that they are likely not to be clinically meaningful.

\section{Disclosure of interest}

All authors declare not to have any conflicts of interest that might be interpreted as influencing the content of the manuscript.

\section{Funding}

This work was supported by the European Union (European Community's Seventh Framework Program [grant number HEALTH-F2-2010-241909; Project EU-GEI]). M.J.K. was supported by a Medical Research Council Fellowship [grant number MR/ J008915/1].

\section{Acknowledgments}

We thank all patients who participated in the study as well as the referring specialists.

\section{Apppendix A}

\section{EU-GEI High Risk Study Group - Author List}

Philip McGuire ${ }^{2}$, Lucia R. Valmaggia ${ }^{3}$, Matthew J. Kempton ${ }^{2}$, Maria Calem ${ }^{2}$, Stefania Tognin ${ }^{2}$, Gemma Modinos ${ }^{2}$, Lieuwe de Haan $^{4,7}$, Mark van der Gaag ${ }^{8,10}$, Eva Velthorst ${ }^{5,11}$, Tamar C. Kraan ${ }^{6}$, Daniella S. van Dam ${ }^{4}$, Nadine Burger ${ }^{7}$, Barnaby Nelson ${ }^{12,13}$, Patrick McGorry $^{12,13}$, G Paul Amminger ${ }^{12,13}$, Christos Pantelis ${ }^{14}$, Athena Politis $^{12,13}$, Joanne Goodall ${ }^{12,13}$, Anita Riecher-Rössler ${ }^{1}$, Stefan Borgwardt $^{1}$, Charlotte Rapp ${ }^{1}$, Sarah Ittig $^{1}$, Erich Studerus ${ }^{1}$, Renata Smieskova $^{1}$, Rodrigo Bressan ${ }^{15}$, Ary Gadelha ${ }^{15}$, Elisa Brietzke ${ }^{16}$, Graccielle Asevedo ${ }^{15}$, Elson Asevedo ${ }^{15}$, Andre Zugman ${ }^{15}$, Neus
Barrantes-Vidal ${ }^{17}$, Tecelli Domínguez-Martínez ${ }^{18}$, Anna Racioppi ${ }^{19}$, Paula Cristóbal-Narváez ${ }^{19}$, Thomas R. Kwapil ${ }^{20}$, Manel Monsonet ${ }^{19}$, Mathilde Kazes ${ }^{21}$, Claire Daban ${ }^{21}$, Julie Bourgin ${ }^{21}$, Olivier Gay ${ }^{21}$, Célia Mam-Lam-Fook ${ }^{21}$, Marie-Odile Krebs ${ }^{21}$, Dorte Nordholm ${ }^{22}$, Lasse Randers ${ }^{22}$, Kristine Krakauer ${ }^{22}$, Louise Glenthøj ${ }^{22}$, Birte Glenthøj ${ }^{23}$, Merete Nordentoft ${ }^{22}$, Stephan Ruhrmann ${ }^{24}$, Dominika Gebhard $^{24}$, Julia Arnhold ${ }^{25}$, Joachim Klosterkötter ${ }^{24}$, Gabriele Sachs $^{26}$, Iris Lasser $^{26}$, Bernadette Winklbaur ${ }^{26}$, Philippe A. Delespaul $^{27,28}$, Bart P. Rutten ${ }^{29}$, and Jim van Os ${ }^{29,30}$

\section{Affiliations}

${ }^{1}$ University Psychiatric Hospital, Wilhelm Klein-Strasse 27, $\mathrm{CH}$ 4002 Basel, Switzerland; ${ }^{2}$ Department of Psychosis Studies, Institute of Psychiatry, Psychology \& Neuroscience, King's College London, De Crespigny Park, Denmark 458 Hill, London, United Kingdom SE5 8AF; 3 Department of Psychology, Institute of Psychiatry, Psychology \& Neuroscience, King's College London, De Crespigny Park, Denmark Hill, 456 London, United Kingdom SE5 8AF; 4 Amsterdam UMC, University of Amsterdam, Psychiatry, Department Early Psychosis, Meibergdreef 9, Amsterdam, The Netherlands; ${ }^{5}$ Department of Psychiatry and Seaver Center for Research and Treatment, Icahn School of Medicine at Mount Sinai, NY, US; 6 Mental Health Institute Arkin, Amsterdam, the Netherlands; ${ }^{7}$ Arkin Amsterdam; ${ }^{8}$ VU University, Faculty of Behavioural and Movement Sciences, Department of Clinical Psychology and Amsterdam Public Mental Health research institute, van der Boechorststraat 7, 1081 BT Amsterdam, The Netherlands; ${ }^{9}$ Mental Health Institute Noord-Holland Noord, Hoorn, the Netherlands; ${ }^{10}$ Parnassia Psychiatric Institute, Department of Psychosis Research, Zoutkeetsingel 40, 2512 HN The Hague, The Netherlands; ${ }^{11}$ Early Psychosis Section, Department of Psychiatry, Academic Medical Centre, University of Amsterdam, Amsterdam, the Netherlands; ${ }^{12}$ Orygen, The National Centre of Excellence in Youth Mental Health, University of Melbourne; ${ }^{13}$ Centre for Youth Mental Health, University of Melbourne, 35 Poplar Road (Locked Bag 10), Parkville, Victoria 485 3052, Australia; 14 Melbourne Neuropsychiatry Centre, The University of Melbourne; 15 LiNC - Lab Interdisciplinar Neurociências Clínicas, Depto Psiquiatria, Escola Paulista de Medicina, Universidade Federal de São Paulo - UNIFESP; ${ }^{16}$ Depto Psiquiatria, Escola Paulista de Medicina, Universidade Federal de São Paulo - UNIFESP; 17 Departament de Psicologia Clínica i de la Salut (Universitat Autònoma de Barcelona), Fundació Sanitària Sant Pere Claver (Spain), Spanish Mental Health Research Network (CIBERSAM); 18 CONACYT-Dirección de Investigaciones Epidemiológicas y Psicosociales, Instituto Nacional de Psiquiatría Ramón de la Fuente Muñiz (México); ${ }^{19}$ Departament de Psicologia Clínica i de la Salut (Universitat Autònoma de Barcelona); ${ }^{20}$ Department of Psychology, University of Illinois at Urbana-Champaign (USA); ${ }^{21}$ University Paris Descartes, Hôpital Sainte-Anne, C'JAAD, Service HospitaloUniversitaire, Inserm U894, Institut de Psychiatrie (CNRS 3557) Paris, France; ${ }^{22}$ Mental Health Center Copenhagen and Center for Clinical Intervention and Neuropsychiatric Schizophrenia Research, CINS, Mental Health Center Glostrup, Mental Health Services in the Capital Region of Copenhagen, University of Copenhagen; ${ }^{23}$ Centre for Neuropsychiatric Schizophrenia Research (CNSR) \& Centre for Clinical Intervention and Neuropsychiatric Schizophrenia Research (CINS), Mental Health Centre Glostrup, University of Copenhagen, Glostrup, Denmark; 24 Department of Psychiatry and Psychotherapy, University of Cologne, Cologne, Germany; ${ }^{25}$ Psyberlin, Berlin, Germany; ${ }^{26}$ Medical University of Vienna, Department of Psychiatry and Psychotherapy; ${ }^{27}$ Department of Psychiatry and Neuropsychology, School for Mental Health and Neuroscience, Maastricht University Medical Centre, P.O. Box 616, 6200 MD 464 Maastricht, The Netherlands; ${ }^{28}$ Mondriaan Mental Health Trust, P.O. Box 4436 CX Heerlen, The Netherlands; 29 Medical University of Vienna, 
Department of Psychiatry and Psychotherapy; 30 Department of Psychosis Studies, Institute of Psychiatry, Psychology \& Neuroscience, King's College London, De Crespigny Park, Denmark 458 Hill, London, United Kingdom SE5 8AF.

\section{References}

[1] Kraepelin E. Dementia praecox and paraphrenia. Huntington, NY: Krieger Publishing Co., Inc.; 19191987.

[2] Riecher-Rössler A, Butler S, Kulkarni J. Sex and gender differences in schizophrenic psychoses - a critical review. Arch Womens Ment Health 2018;21:627-48.

[3] Häfner H, Riecher-Rössler A, Fätkenheuer B, Hambrecht M, Löffler W, An der Heiden W. Sex differences in schizophrenia. Psychiatr Fenn 1991;22:123-56.

[4] Häfner H, Maurer K, Löffler W, Riecher-Rössler A. The influence of age and sex on the onset and early course of schizophrenia. Br J Psychiatry 1993;162:80-6.

[5] Riecher-Rössler A, McGorry P. Early detection and intervention in psychosis. State of the art and future perspectives. Key issues in mental health ed, Vol. 181. Basel: Karger; 2016.

[6] Barajas A, Ochoa S, Obiols JE, Lalucat-Jo L. Gender differences in individuals at high-risk of psychosis: a comprehensive literature review. Sci World J 2015;2015:430735.

[7] Seedat S, Scott KM, Angermeyer MC, Berglund P, Bromet EJ, Brugha TS, et al. Cross-national associations between gender and mental disorders in the World Health Organization World Mental Health Surveys. Arch Gen Psychiatry 2009;66:785-95.

[8] Albert U, Tomassi S, Maina G, Tosato S. Prevalence of non-psychotic disorders in ultra-high risk individuals and transition to psychosis: a systematic review. Psychiatry Res 2018;270:1-12.

[9] Fusar-Poli P, Nelson B, Valmaggia L, Yung AR, McGuire PK. Comorbid depressive and anxiety disorders in 509 individuals with an at-risk mental state: impact on psychopathology and transition to psychosis. Schizophr Bull 2014;40:120-31.

[10] Kline ER, Seidman LJ, Cornblatt BA, Woodberry KA, Bryant C, Bearden CE, et al Depression and clinical high-risk states: baseline presentation of depressed vs. non-depressed participants in the NAPLS-2 cohort. Schizophr Res 2018;192:357-63.

[11] Rietschel L, Lambert M, Karow A, Zink M, Müller H, Heinz A, et al. Clinical high risk for psychosis: gender differences in symptoms and social functioning. Early Interv Psychiatry 2015, doi:http://dx.doi.org/10.1111/eip.12240.

[12] Kraan TC, Velthorst E, Themmen M, Valmaggia L, Kempton MJ, McGuire P, et al. Child maltreatment and clinical outcome in individuals at ultra-high risk for psychosis in the EU-GEI High Risk Study. Schizophr Bull 2018;44: 584-92.

[13] Yung AR, Yuen HP, McGorry PD, Philips LJ, Kelly D, Dell'olio M, et al. Mapping the onset of psychosis: the comprehensive assessment of at-risk mental states. Aust N Z J Psychiatry 2005;39:964-71.

[14] Wittchen H-U, Zauding M, Fydrich T. Strukturiertes klinisches interview für DSM-IV. Achse-I: psychische Störungen. Göttingen. Hogrefe; 1997.

[15] Mallett R. Sociodemographic schedule. London: Sectionof Social Psychiatry, Institute of Psychiatry; 1997.

[16] Shafer A, Dazzi F, Ventura J. Factor structure of the brief psychiatric rating scale - expanded (BPRS-E) in a large hospitalized sample. J Psychiatr Res 2017;93:79-86.
[17] Andreasen NC. Scale for the assessment of negative symptoms (SANS). Br J Psychiatry 1989;155:53-8.

[18] Montgomery SA, Asberg M. A new depression scale designed to be sensitive to change. Br J Psychiatry 1979:134:382-9.

[19] Young RC, Biggs JT, Ziegler VE, Meyer DA. A rating scale for mania: reliability, validity and sensitivity. B J Psychiatry 1978;133:429-35.

[20] Miyakoshi T, Matsumoto K, Ito F, Ohmuro N, Matsuoka H. Application of the Comprehensive Assessment of At-Risk Mental States (CAARMS) to the Japanese population: reliabiliy and validity of the Japanese version of the CAARMS. Early Interv Psychiatry 2009;3:123-30.

[21] Quilty L, Robinson J, Rolland J-P, De Fruyt F, Rouillon F, Bagby R. The structure of the Montgomery-Asberg depression rating scale over the course of treatment for depression. Int J Methods Psychiatr Res 2013;22:175-84.

[22] Barkus EJ, Stirling J, Hopkins RS, Lewis S. Cannabis-induced psychosis-like experiences are associated with high schizotypy. Psychopathology 2006;39:175-8.

[23] Goldman HH, Skodol AE, Lave TR. Revising axis V for DSM-IV: a review of measures of social functioning. Am J Psychiatry 1992;149:1148-56.

[24] Team RC. R: a language and environment for statistical computing. 2017.

[25] Benjamini Y, Hochberg Y. Controlling the false discovery rate - a practival and powerful approach to multiple testing. J R Stat Soc Ser B 1995;289-300.

[26] Gonzalez-Rodriguez A, Studerus E, Spitz A, Bugra H, Aston J, Borgwardt S, et al. Gender differences in the psychopathology of emerging psychosis. Isr J Psychiatry Relat Sci 2014;51:85-92.

[27] Willhite RK, Niendam TA, Bearden CE, Zinberg J, O’Brien MP, Cannon TD. Gender differences in symptoms, functioning and social support in patients at ultra-high risk for developing a psychotic disorder. Schizophr Res 2008;104:237-45

[28] Rietdijk J, Ising HK, Dragt S, Klaassen R, Nieman D, Wunderink L, et al. Depression and social anxiety in help-seeking patients with an ultra-high risk for developing psychosis. Psychiatry Res 2013;209:309-13.

[29] Pruessner M, Faridi K, Shah J, Rabinovitch M, Iyer S, Abadi S, et al. The clinic for assessment of youth at risk (CAYR): 10 years of service delivery and research targeting the prevention of psychosis in Montreal, Canada. Early Interv Psychiatry 2017;11:177-84.

[30] McLean CP, Asnaani A, Litz BT, Hofmann SG. Gender differences in anxiety disorders: prevalence, course of illness, comorbidity and burden of illness. J Psychiatr Res 2011;45:1027-35.

[31] Riecher-Rössler A, Pflueger MO, Borgwardt S. Schizophrenia in women. In: Kohen D, editor. Oxford textbook of women and mental health. Oxford: Oxford University Press; 2010. p. 102-14.

[32] Riecher-Rössler A. Prospects for the classification of mental disorders in women. Eur Psychiatry 2010;25:189-96.

[33] Wilson RP, Patel R, Bhattacharyya S. Do fewer males present to clinical highrisk services for psychosis relative to first-episode services? Early Interv Psychiatry 2017:11:429-35.

[34] Fusar-Poli P, Borgwardt S, Bechdolf A, Addington J, Riecher-Rössler A, SchultzeLutter F, et al. The psychosis high-risk state: a comprehensive state-of-the art review. JAMA Psychiat 2013;70(1):107-20.

[35] Riecher-Rössler A, Studerus E. Prediction of conversion to psychosis in individuals with an at-risk mental state: a brief update on recent developments. Curr Opin Psychiatry 2017;30:209-19.

[36] Beck K, Andreou C, Studerus E, Heitz U, Ittig S, Leanza L, et al. Clinical and functional long-term outcome of patients at clinical high risk (CHR) for psychosis without transition to psychosis: a systematic review (in press). Schizophr Res 2019, doi:http://dx.doi.org/10.1016/ j.schres.2018.12.047. 